Review Article

\title{
Effects of Nutritional Strategies on Glucose Homeostasis in Gestational Diabetes Mellitus: A Systematic Review and Network Meta-Analysis
}

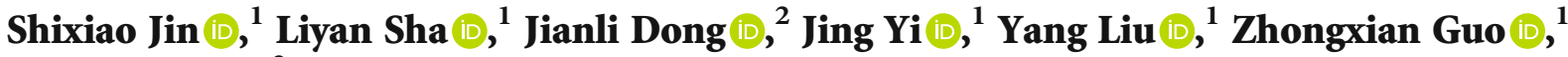 \\ and Bing $\mathrm{Hu}$ iD $^{3}$ \\ ${ }^{1}$ Department of Nursing, The Second Affiliated Hospital of Dalian Medical University, Dalian 116000, China \\ ${ }^{2}$ Department of International Medicine, The Second Affiliated Hospital of Dalian Medical University, Dalian 116000, China \\ ${ }^{3}$ Department of Gynaecology and Obstetrics, The Second Affiliated Hospital of Dalian Medical University, Dalian 116000, China \\ Correspondence should be addressed to Liyan Sha; slydl2007@163.com
}

Received 8 November 2019; Accepted 3 February 2020; Published 25 February 2020

Academic Editor: Viral Shah

Copyright () 2020 Shixiao Jin et al. This is an open access article distributed under the Creative Commons Attribution License, which permits unrestricted use, distribution, and reproduction in any medium, provided the original work is properly cited.

\begin{abstract}
Background. Gestational diabetes mellitus (GDM) is one of the most common complications of pregnancy, and nutritional therapy is the basis of GDM treatment. However, the effects of different forms of nutritional supplementation on improving gestational diabetes are uncertain. Objective. We conducted a network meta-analysis to evaluate the effects of supplementation with different nutrients on glucose metabolism in women with GDM. Methods. We conducted a literature search using PubMed, EMBASE, and the Cochrane Library to identify randomized controlled trials (RCTs) comparing the differences between different nutritional strategies in women with GDM. The Cochrane tool was used to assess the risk of bias. Pairwise metaanalysis and network meta-analysis were used to compare and rank the effects of nutritional strategies for the improvement of fasting plasma glucose (FPG), serum insulin, and homeostasis model assessment-insulin resistance (HOMA-IR). Results. We included thirteen RCTs with a total of 754 participants. Compared with placebo, omega-3, magnesium, vitamin D, zinc, and probiotics were more beneficial for improving FPG, serum insulin, and HOMA-IR. Network analysis showed that vitamin D supplementation was superior to omega-3 (-3.64 mg/dL, 95\% CI: -5.77 to -1.51$)$, zinc $(-5.71 \mathrm{mg} / \mathrm{dL}, 95 \%$ CI: -10.19 to -1.23$)$, probiotics $(-6.76 \mathrm{mg} / \mathrm{dL}, 95 \% \mathrm{CI}:-10.02$ to -3.50$)$, and placebo $(-12.13 \mathrm{mg} / \mathrm{dL}, 95 \% \mathrm{CI}$ : -14.55 to -9.70$)$ for improving FPG. Magnesium supplementation was more beneficial for decreasing serum insulin compared with probiotics $(-5.10 \mu \mathrm{IU} / \mathrm{mL}, 95 \%$ CI: -9.32 to -0.88$)$ and placebo $(-7.80 \mu \mathrm{IU} / \mathrm{mL} ; 95 \%$ CI-11.95, -3.65). Vitamin D was more effective than probiotics $(-0.99,95 \%$ CI: -1.84 to -0.14$)$ and placebo $(-1.80,95 \% \mathrm{CI}$ : -2.45 to -1.16$)$ for improving HOMA-IR. Conclusion. Vitamin D supplementation significantly reduced FPG and regulated HOMA-IR. Magnesium supplementation was superior in decreasing serum insulin than supplementation with other nutrients. Nutrient supplementation seemed to have an effect on glucose homeostasis maintenance in patients with GDM and may be considered an adjunctive therapy.
\end{abstract}

\section{Introduction}

Gestational diabetes mellitus (GDM) is defined as any degree of glucose intolerance with an onset or first recognition during pregnancy [1] and is one of the most common complications during pregnancy. GDM affects $9.3 \%-25.5 \%$ of pregnant women, and its incidence is continuing to increase [2]. GDM is considered to be a form of impaired glucose tolerance similar to prediabetes in nonpregnant individuals and has become a global public health problem that is associated with short-term and long-term adverse health problems in mothers and their offspring [3]. Severe GDM increases the risk of spontaneous abortion and preeclampsia during pregnancy and can lead to the occurrence of congenital abnormalities, fetal macrosomia, and hypoglycemia in newborns $[4,5]$. Studies have shown that GDM is a major risk factor for the development of impaired glucose tolerance and type 2 diabetes mellitus among pregnant women; women 
develop diabetes mellitus at rates of $20-60 \%$ within 5-10 years after pregnancy $[6,7]$. The risks of developing metabolic syndrome and cardiovascular disease are three times higher in women with GDM. In addition, children born to women with GDM have increased risks of impaired glucose tolerance and obesity.

GDM is thought to be related to the progression of pregnancy; islet resistance is affected by hormones, leading to decreased insulin sensitivity and glucose intolerance [8]. Therefore, it is essential to identify reasonable and effective methods for improving insulin sensitivity and maintaining blood glucose homeostasis. Dietary therapy is considered to be the first-line treatment for GDM [9]. The purpose is to strictly control the glucose levels of pregnant women on the basis of conventional treatment, ensure the reasonable nutritional intake of pregnant women and fetuses, and reduce the occurrence of complications in pregnant women and their children. The International Federation of Obstetrics and Gynecology (FIGO), the American Diabetes Association (ADA), the Canadian Diabetes Association (CDA), and the Japanese Diabetes Association (JDS) expounded the importance of diet for the treatment of gestational diabetes [10-13]. Strictly controlled blood glucose during pregnancy has been shown to reduce the risk of complications; however, at present, no clear guideline for GDM dietary management exists. The dietary advice for GDM patients has focused on limiting carbohydrates [14], and there is still no consensus on the best nutritional strategies for improving blood glucose. The challenge is now to determine which nutritional strategies are reasonable and effective for GDM. Therefore, this study is aimed at evaluating the effectiveness of different nutritional strategies on blood glucose homeostasis in GDM patients and ranking the effectiveness of providing safe and effective methods for the management of GDM.

\section{Materials and Methods}

The study was conducted according to the Preferred Reporting Items for Systematic Reviews and Meta-Analyses (PRISMA) extension statement for reporting network metaanalyses of health care interventions [15].

2.1. Search Strategy. The PubMed, EMBASE, and Cochrane Library electronic databases were searched for randomized controlled trials (RCTs) that evaluated GDM and nutritional strategies published between database inception and July 2019 using the following terms: ('diabetes, gestational' or 'pregnancy-induced diabetes' or 'gestational diabetes mellitus' or 'GDM') AND ('nutrient"' or 'nutrition' or 'dietary supplement' or 'protein' or 'amino acids' or 'fatty acids' or 'vitamin' or 'mineral' or 'antioxidant' or 'phytochemical') AND ('randomized controlled trial' or 'controlled trial' or 'clinical trial' or 'random*' or 'RCT'). We also scanned the reference lists of the retrieved articles to identify additional eligible studies. Annex 1 of the supplemental material details the search strategy.

2.2. Eligibility Criteria. The eligibility criteria are detailed below following the participants, intervention, controls, out- comes and study design (PICOS) framework [16]: (i) Participants: we included studies enrolling participants with GDM. (ii) Interventions: any RCTs evaluating nutrient supplementation compared with placebo on the basis of receiving nutritional treatment or maintaining the usual diets were included. (iii) Controls: groups receiving placebo or those receiving any nutrient supplementation on the basis of receiving nutritional treatment or maintaining the usual diet were considered. Studies without control conditions were excluded. (iv) Outcomes: the outcome measures were changes in fasting plasma glucose (FPG), serum insulin, and homeostasis model assessment-insulin resistance (HOMA-IR). (v) Studies: only RCTs were considered.

2.3. Data Extraction. All retrieved articles were combined in EndnoteX7 to remove duplicates. Two researchers independently screened the titles and abstracts according to the prespecified criteria. The full texts of articles that potentially met the eligibility criteria were reviewed. In cases of disagreement, a third researcher was consulted for a final decision. The two researchers independently extracted and crosschecked the data. For each included study, the following data were extracted: general information, study characteristics, interventions, and outcomes.

2.4. Quality Assessment. Cochrane's risk of bias tool was used by two researchers to independently assess the risk of bias [17], including the following: random sequence generation, allocation concealment, blinding of participants and personnel, blinding of outcome assessment, incomplete outcome data, selective reporting, and other bias. Each quality assessment was classified as low risk of bias, high risk of bias, or unclear (moderate risk of bias).

2.5. Statistical Analysis. We performed a pairwise metaanalysis using the random effects model for every intervention comparison, and the $I^{2}$ statistic and $P$ values were calculated as a measure of the statistical heterogeneity [18], with $I^{2} \geq 50 \%$ indicating substantial heterogeneity.

In addition, network meta-analysis (NMA) was conducted to estimate the comparative effects of different types of nutrient supplementation on maintaining glucose homeostasis. The results of the comparative effects are presented as the weighted mean differences (WMDs) and 95\% confidence intervals (CIs). We also estimated the ranking probabilities of the intervention effect using surface under the cumulative ranking curve (SUCRA) [19]. The larger the SUCRA value, the better the ranking of the intervention effect. Transitivity is the basis of NMA [20]. The consistency between the direct and indirect evidence was evaluated using inconsistency tests to assess the validity of the transitivity assumption. Publication biases or small sample effects were examined using a comparison-adjusted funnel plot.

Review Manager version 5.3 and Stata version 15 were used to conduct the analyses, and the extracted data were subjected to in-depth analysis [21]. The "metan" package was used for the pairwise meta-analysis, and the "network" package was used to conduct the NMA. Statistical significance was set as a $P$ value $<0.05$ in all analyses. 


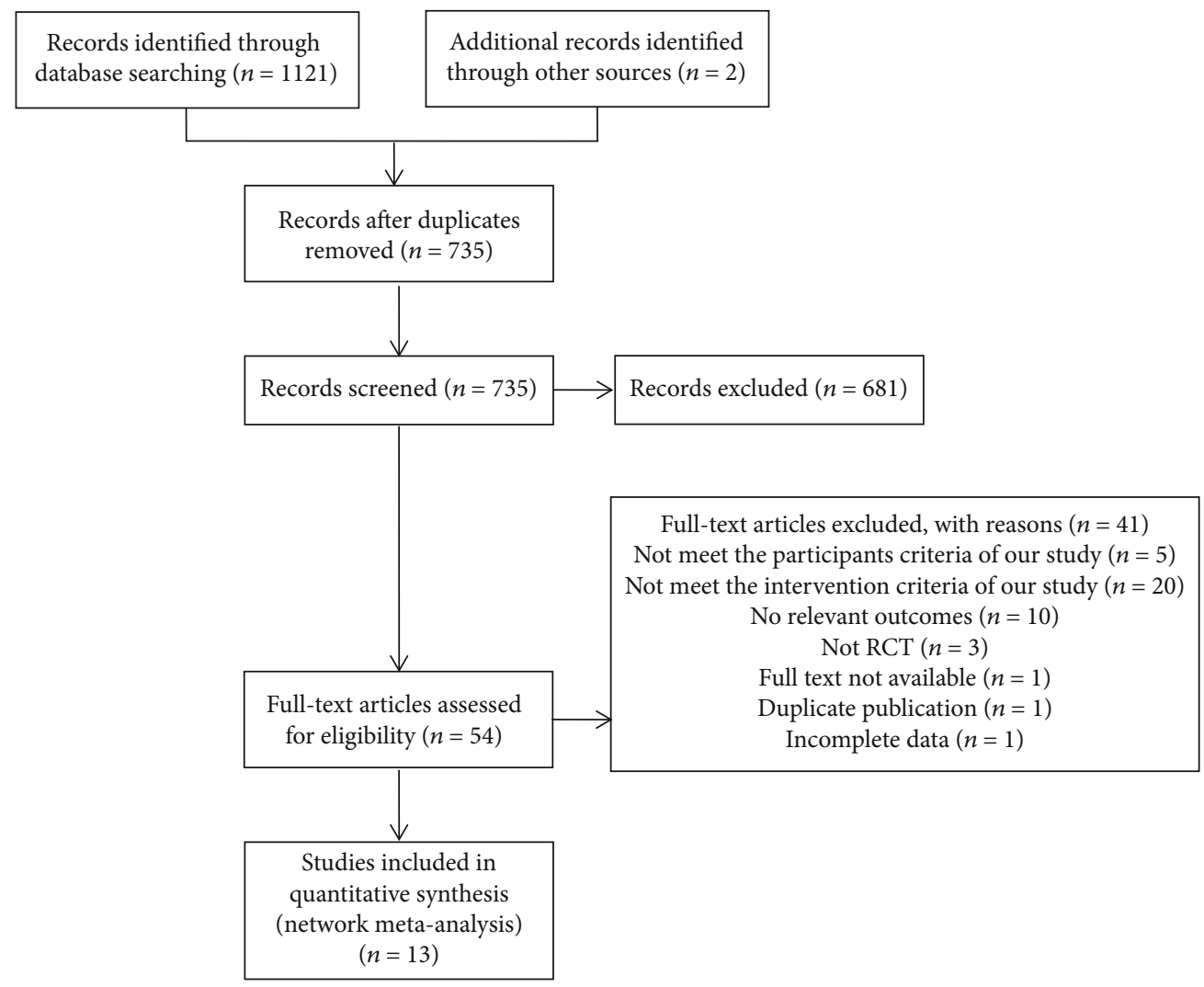

Figure 1: Study flow diagram.

\section{Results}

3.1. Study Selection and Characteristics. A total of 1121 articles were retrieved by following the predesigned literature retrieval strategy. By further searching the references included in the articles, 2 additional articles were obtained. After reading the titles and abstracts, 54 studies were selected for further review. Finally, thirteen studies met our inclusion criteria [22-34]. The detailed process of the search strategy is described in Figure 1. Table 1 summarizes the basic characteristics of the included studies. The included studies were published between 2013 and 2019, and eleven trials were from Iran, while the other two were from China and Thailand. A total of 754 participants were included in this review and the average age of the participants was 29.8 years. The mean duration of the intervention was 6.8 weeks. Five studies including 310 participants mentioned mean gestational age, and the mean gestational age of these participants was 22.4 weeks.

3.2. Results of Risk of Bias. Figure 2 shows the risk of bias of the included studies. Twelve studies [22-29, 31-34] reported methods for generating random sequences, which were mainly computerized randomization methods. Seven studies mentioned the use of allocation concealment, and three of these studies described specific methods [25, 27, 29]. Five studies [22, 25, 28, 31, 33] clearly illustrated the blinding of participants and personnel. Two studies $[25,27]$ indicated that the outcome assessors were blinded. Six studies [23-25,
$27,29,33$ ] clarified that the analytical method was based on intention-to-treat analysis.

3.3. Pairwise Meta-Analysis. We used paired meta-analysis to analyze the effects of nutritional interventions on glucose homeostasis in GDM women from three aspects, including FPG, insulin, and HOMA-IR. The results showed that nutritional supplementation was effective in regulating FPG, insulin, and HOMA-IR compared with a placebo. Five comparisons (placebo vs. omega-3, placebo vs. magnesium, placebo vs. vitamin $\mathrm{D}$, and placebo vs. zinc in regulating FPG and placebo vs. probiotics in regulating insulin) showed no heterogeneity. Regarding the regulation of insulin and HOMA-IR, the comparison of placebo vs. zinc showed high heterogeneity $\left(I^{2}=62 \%\right.$ and $I^{2}=81 \%$, respectively). Table 2 displays the detailed results. In terms of the source of heterogeneity, the meta-analysis included only two studies, and the study by Roshanravan et al. [30], which did not mention information on allocation concealment or the blinding of the outcome assessment, may have led to higher heterogeneity in the study.

3.4. Network Plots. Figure 3 shows the network plots of the included studies. We included six interventions in the network meta-analysis: omega-3, magnesium, vitamin $\mathrm{D}$, zinc, probiotic, and placebo. Each point represents an intervention, and the size of the point represents the sample size of the intervention. Lines between points represent the direct 


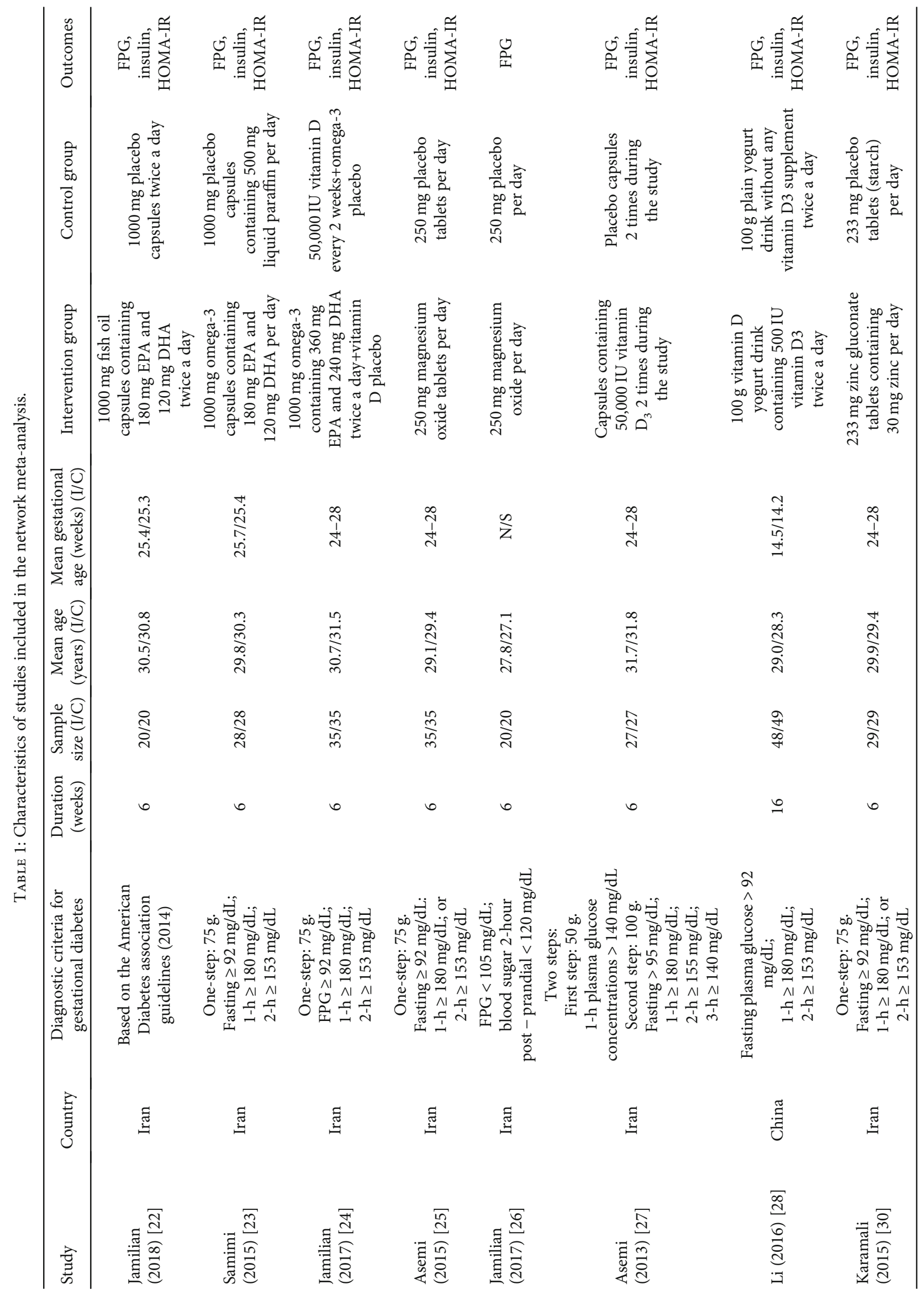




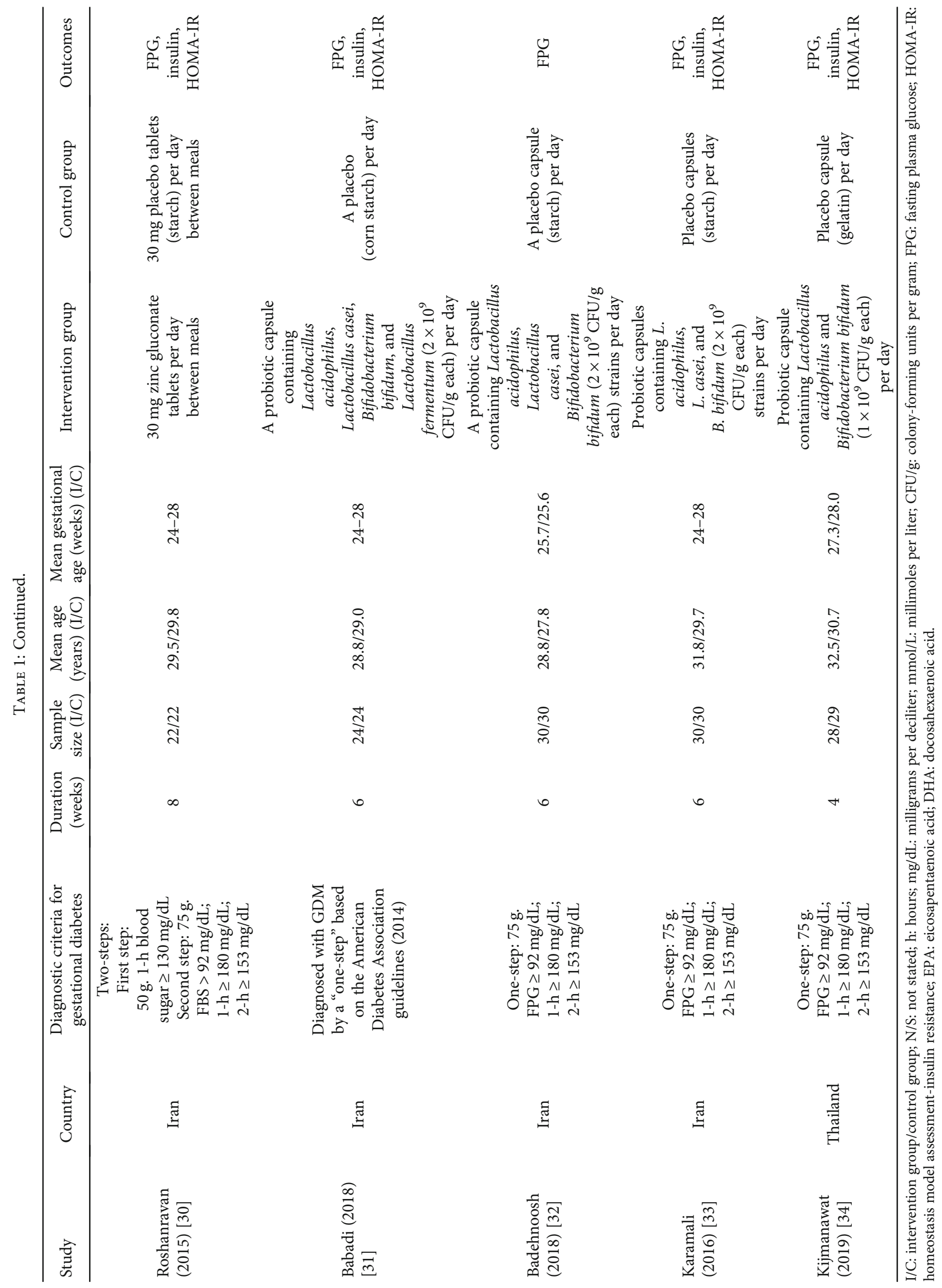




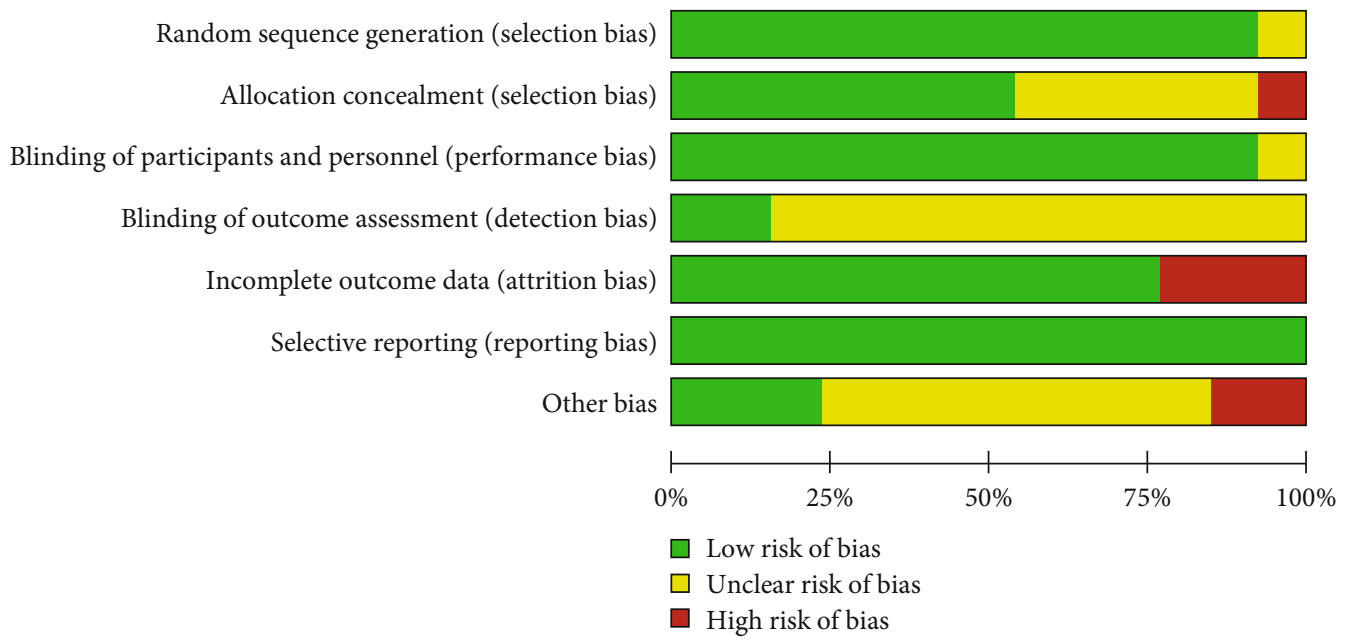

FIGURE 2: Risk of bias in the included studies.

TABLE 2: Results of pairwise meta-analysis.

\begin{tabular}{|c|c|c|c|c|}
\hline & Studies & Patients & WMD $(95 \% \mathrm{CI})$ & $I^{2}$ \\
\hline \multicolumn{5}{|l|}{ FPG } \\
\hline Placebo vs. omega-3 & 2 & 96 & $-5.93(-10.29,-1.57)$ & $0 \%$ \\
\hline Placebo vs. magnesium & 2 & 110 & $-10.59(-13.68,-7.50)$ & $0 \%$ \\
\hline Placebo vs. vitamin D & 2 & 151 & $-13.17(-15.95,-10.39)$ & $0 \%$ \\
\hline Placebo vs. zinc & 2 & 102 & $-6.42(-10.18,-2.65)$ & $0 \%$ \\
\hline Placebo vs. probiotic & 4 & 225 & $-5.49(-8.05,-2.93)$ & $25 \%$ \\
\hline \multicolumn{5}{|l|}{ Insulin } \\
\hline Placebo vs. omega-3 & 2 & 96 & $-3.22(-6.21,-0.24)$ & $28 \%$ \\
\hline Placebo vs. vitamin D & 2 & 151 & $-6.23(-8.05,-4.40)$ & $29 \%$ \\
\hline Placebo vs. zinc & 2 & 102 & $-4.61(-7.04,-2.18)$ & $62 \%$ \\
\hline Placebo vs. probiotic & 3 & 165 & $-2.70(-3.46,-1.94)$ & $0 \%$ \\
\hline \multicolumn{5}{|l|}{ HOMA-IR } \\
\hline Placebo vs. omega-3 & 2 & 96 & $-1.01(-1.81,-0.21)$ & $17 \%$ \\
\hline Placebo vs. vitamin D & 2 & 151 & $-1.97(-2.51,-1.42)$ & $5 \%$ \\
\hline Placebo vs. zinc & 2 & 102 & $-0.97(-1.70,-0.23)$ & $81 \%$ \\
\hline Placebo vs. probiotic & 3 & 165 & $-0.69(-0.88,-0.50)$ & $33 \%$ \\
\hline
\end{tabular}

comparison evidence, and the number of studies is reflected by the thickness of the line.

3.5. Results of Network Meta-Analysis. The inconsistency test showed that FPG $\left(\chi^{2}=2.24 ; P=0.1341\right)$, insulin $\left(\chi^{2}=0.81\right.$; $P=0.3678)$, and HOMA-IR $\left(\chi^{2}=056 ; P=0.4532\right)$ exhibited no inconsistencies in the global analysis at the levels of $P$ value $>0.05$, indicating that the direct comparison and indirect comparison results were consistent.

3.5.1. FPG. Table 3 shows the results of the effects of the interventions on FPG. Thirteen studies reported the impact of different interventions on FPG control. Compared with placebo, vitamin D (-12.13 $\mathrm{mg} / \mathrm{dL}, 95 \% \mathrm{CI}:-14.55$ to -9.70$)$, magnesium $(-10.59 \mathrm{mg} / \mathrm{dL}, 95 \% \mathrm{CI}:-13.68$ to -7.50$)$, omega-3 $(-8.49 \mathrm{mg} / \mathrm{dL}, 95 \% \mathrm{CI}:-11.28$ to -5.70$)$, zinc $(-6.42 \mathrm{mg} / \mathrm{dL}, 95 \% \mathrm{CI}:-10.18$ to -2.65$)$, and probiotics
$(-5.37 \mathrm{mg} / \mathrm{dL}, 95 \% \mathrm{CI}:-7.54$ to -3.19$)$ resulted in a significant reduction in FPG. Compared to probiotics, vitamin D $(-6.76 \mathrm{mg} / \mathrm{dL}, 95 \% \mathrm{CI}:-10.02$ to -3.50$)$ and magnesium $(-5.22 \mathrm{mg} / \mathrm{dL}, 95 \% \mathrm{CI}:-9.00$ to -1.44$)$ resulted in a greater reduction in FPG. Compared to omega-3 and zinc, vitamin D $(-3.64 \mathrm{mg} / \mathrm{dL}, 95 \% \mathrm{CI}:-5.77$ to $-1.51 ;-5.71 \mathrm{mg} / \mathrm{dL}, 95 \%$ CI: -10.19 to -1.23$)$ was more effective in reducing FPG. There were no significant differences between the other interventions in terms of the effectiveness in reducing FPG.

3.5.2. Insulin. Table 4 shows the results of the effects of the interventions on insulin. Eleven studies on insulin regulation were included in our review. Compared to placebo, magnesium $(-7.80 \mu \mathrm{IU} / \mathrm{mL}, 95 \% \mathrm{CI}:-11.95$ to -3.65$)$, vitamin $\mathrm{D}$ $(-5.89 \mu \mathrm{IU} / \mathrm{mL}, 95 \% \mathrm{CI}:-7.57$ to -4.22$)$, zinc $(-4.78 \mu \mathrm{IU} / \mathrm{mL}$, 95\% CI: -8.16 to -1.41$)$, omega-3 $(-4.12 \mu \mathrm{IU} / \mathrm{mL}, 95 \% \mathrm{CI}$ : -6.38 to -1.86$)$, and probiotics $(-2.70 \mu \mathrm{IU} / \mathrm{mL}, 95 \% \mathrm{CI}:-3.46$ 


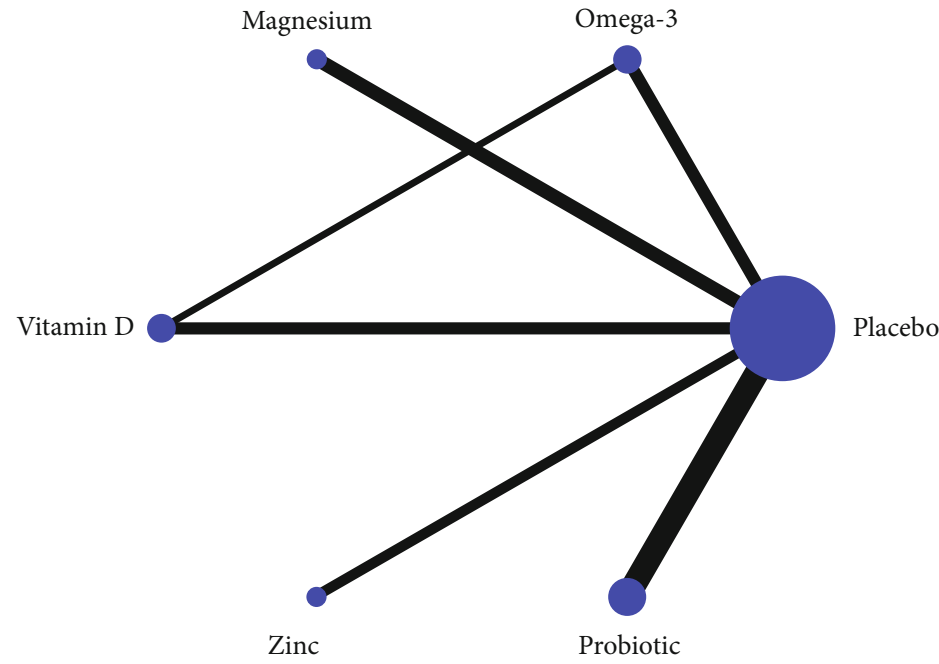

FIGURE 3: Network plots of eligible comparisons for different nutritional strategies.

TABLE 3: Results of the network meta-analysis on FPG.

\begin{tabular}{|c|c|c|c|c|c|}
\hline Magnesium & $2.10(-2.07,6.26)$ & $10.59(7.50,13.68)$ & $5.22(1.44,9.00)$ & $-1.54(-5.47,2.39)$ & $4.17(-0.70,9.04)$ \\
\hline$-2.10(-6.26,2.07)$ & Omega-3 & $8.49(5.70,11.28)$ & $3.12(-0.42,6.66)$ & $-3.64(-5.77,-1.51)$ & $2.07(-2.61,6.76)$ \\
\hline$-10.59(-13.68,-7.50)$ & $-8.49(-11.28,-5.70)$ & Placebo & $-5.37(-7.54,-3.19)$ & $-12.13(-14.55,-9.70)$ & $-6.42(-10.18,-2.65)$ \\
\hline$-5.22(-9.00,-1.44)$ & $-3.12(-6.66,0.42)$ & $5.37(3.19,7.54)$ & Probiotics & $-6.76(-10.02,-3.50)$ & $-1.05(-5.40,3.30)$ \\
\hline $1.54(-2.39,5.47)$ & $3.64(1.51,5.77)$ & $12.13(9.70,14.55)$ & $6.76(3.50,10.02)$ & Vitamin D & $5.71(1.23,10.19)$ \\
\hline$-4.17(-9.04,0.70)$ & $-2.07(-6.76,2.61)$ & $6.42(2.65,10.18)$ & $1.05(-3.30,5.40)$ & $-5.71(-10.19,-1.23)$ & Zinc \\
\hline
\end{tabular}

Comparing the effects (weighted mean differences: WMDs) of all nutritional strategies and 95\% confidence intervals (95\% CIs). Significant results are shown in bold.

TABLE 4: Results of the network meta-analysis on insulin.

\begin{tabular}{|c|c|c|c|c|c|}
\hline Magnesium & $3.68(-1.05,8.41)$ & $7.80(3.65,11.95)$ & $5.10(0.88,9.32)$ & $1.91(-2.57,6.38)$ & $3.02(-2.33,8.36)$ \\
\hline$-3.68(-8.41,1.05)$ & Omega-3 & $4.12(1.86,6.38)$ & $1.42(-0.97,3.81)$ & $-1.77(-4.03,0.48)$ & $-0.66(-4.73,3.40)$ \\
\hline$-7.80(-11.95,-3.65)$ & $-4.12(-6.38,-1.86)$ & Placebo & $-2.70(-3.46,-1.94)$ & $-5.89(-7.57,-4.22)$ & $-4.78(-8.16,-1.41)$ \\
\hline$-5.10(-9.32,-0.88)$ & $-1.42(-3.81,0.97)$ & $2.70(1.94,3.46)$ & Probiotics & $-3.19(-5.03,-1.36)$ & $-2.08(-5.55,1.38)$ \\
\hline$-1.91(-6.38,2.57)$ & $1.77(-0.48,4.03)$ & $5.89(4.22,7.57)$ & $3.19(1.36,5.03)$ & Vitamin D & $1.11(-2.66,4.88)$ \\
\hline$-3.02(-8.36,2.33)$ & $0.66(-3.40,4.73)$ & $4.78(1.41,8.16)$ & $2.08(-1.38,5.55)$ & $-1.11(-4.88,2.66)$ & Zinc \\
\hline
\end{tabular}

Comparing the effects (weighted mean differences: WMDs) of all nutritional strategies and 95\% confidence intervals (95\% CIs). Significant results are shown in bold.

to -1.94$)$ resulted in a significant reduction in insulin. Compared to probiotics, magnesium $(-5.10 \mu \mathrm{IU} / \mathrm{mL}, 95 \% \mathrm{CI}$ : -9.32 to -0.88$)$ and vitamin $\mathrm{D}(-3.19 \mu \mathrm{IU} / \mathrm{mL}, 95 \% \mathrm{CI}:-5.03$ to -1.36$)$ resulted in a greater reduction in insulin. There were no significant differences between the effectiveness of the other interventions in terms of insulin reduction.

3.5.3. HOMA-IR. Table 5 shows the results of the effects of the interventions on HOMA-IR. Eleven studies reported HOMA-IR and were included in our NMA. Compared with placebo, vitamin D (-1.80, 95\% CI: -2.45 to -1.16$)$, magnesium $(-1.90,95 \% \mathrm{CI}:-3.01$ to -0.79$)$, omega-3 $(1.25,95 \%$ CI: -2.00 to 0.51$)$, zinc $(-1.01,95 \% \mathrm{CI}:-1.90$ to -0.12$)$, and probiotics $(-0.81,95 \% \mathrm{CI}:-1.35$ to -0.28$)$ showed a greater benefit in improving HOMA-IR. Compared to probiotics, vitamin D ( $-0.99,95 \% \mathrm{CI}:-1.84$ to -0.14$)$ was more effective in improving HOMA-IR. There were no significant differences between the effectiveness of the other interventions in improving HOMA-IR.

3.6. Rank Probabilities. The NMA can estimate the best effects of each intervention on different outcomes and rank each nutritional supplementation based on SUCRA values. The larger SUCRA values indicate a better effect of intervention. Table 6 and Figure 4 show the detailed results of the ranking. The ranking of the effectiveness of each intervention for different outcomes showed that vitamin D supplementation was the most beneficial nutritional strategy for 
TABLE 5: Results of the network meta-analysis on HOMA-IR.

\begin{tabular}{|c|c|c|c|c|c|}
\hline Magnesium & $0.65(-0.69,1.98)$ & $1.90(0.79,3.01)$ & $1.09(-0.14,2.32)$ & $0.10(-1.19,1.38)$ & $0.89(-0.53,2.31)$ \\
\hline$-0.65(-1.98,0.69)$ & Omega-3 & $1.25(0.51,2.00)$ & $0.44(-0.50,1.39)$ & $-0.55(-1.30,0.21)$ & $0.25(-0.93,1.42)$ \\
\hline$-1.90(-3.01,-0.79)$ & $-1.25(-2.00,-0.51)$ & Placebo & $-0.81(-1.35,-0.28)$ & $-1.80(-2.45,-1.16)$ & $-1.01(-1.90,-0.12)$ \\
\hline$-1.09(-2.32,0.14)$ & $-0.44(-1.39,0.50)$ & $0.81(0.28,1.35)$ & Probiotics & $-0.99(-1.84,-0.14)$ & $-0.20(-1.21,0.82)$ \\
\hline$-0.10(-1.38,1.19)$ & $0.55(-0.21,1.30)$ & $1.80(1.16,2.45)$ & $0.99(0.14,1.84)$ & Vitamin D & $0.79(-0.31,1.90)$ \\
\hline$-0.89(-2.31,0.53)$ & $-0.25(-1.42,0.93)$ & $1.01(0.12,1.90)$ & $0.20(-0.82,1.21)$ & $-0.79(-1.90,0.31)$ & Zinc \\
\hline
\end{tabular}

Comparing the effects (weighted mean differences: WMDs) of all nutritional strategies and 95\% confidence intervals (95\% CIs). Significant results are shown in bold.

TABLE 6: Ranking results of the comparative effects of different nutritional strategies on the maintenance of glucose homeostasis.

\begin{tabular}{|c|c|c|c|c|c|c|}
\hline \multirow[t]{2}{*}{ Treatments } & \multicolumn{2}{|c|}{ FPG } & \multicolumn{2}{|c|}{ Insulin } & \multicolumn{2}{|c|}{ HOMA-IR } \\
\hline & SUCRA (\%) & Mean rank & SUCRA (\%) & Mean rank & SUCRA (\%) & Mean rank \\
\hline Placebo & 0 & 6.0 & 0.1 & 6.0 & 0.3 & 6.0 \\
\hline Omega-3 & 58.5 & 3.1 & 47.3 & 3.6 & 55.0 & 3.3 \\
\hline Magnesium & 80.0 & 2.0 & 92.0 & 1.4 & 84.2 & 1.8 \\
\hline Vitamin D & 95.5 & 1.2 & 77.5 & 2.1 & 85.5 & 1.7 \\
\hline Zinc & 38.6 & 4.1 & 58.2 & 3.1 & 43.3 & 3.8 \\
\hline Probiotics & 27.3 & 4.6 & 25.0 & 4.8 & 31.6 & 4.4 \\
\hline
\end{tabular}

FPG: fasting plasma glucose; HOMA-IR: homeostasis model assessment-insulin resistance.
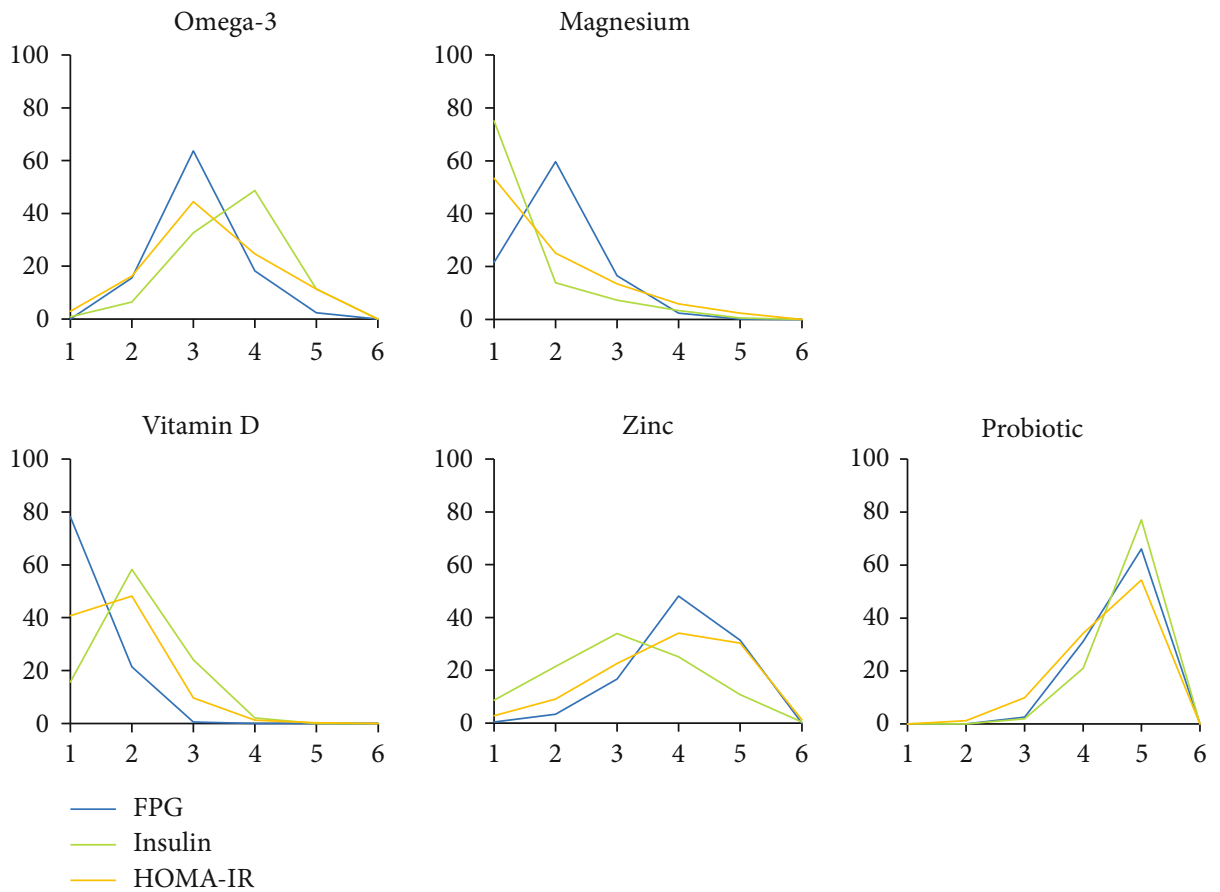

FIGURE 4: Results of different nutritional strategies ranked on the maintenance of glucose homeostasis. Lines of different colors represent different outcomes.

controlling FPG and improving HOMA-IR. The most effective nutritional strategy for reducing insulin concentration was magnesium supplementation.

3.7. Comparison-Adjusted Funnel Plot. Figure 5 shows a comparison-adjusted funnel plot. All studies on the funnel plot were symmetrically distributed on either side of the ver- tical line of $X=0$, indicating that there were no significant small sample effects or publication bias.

\section{Discussion}

GDM is a special type of diabetes mellitus that can cause adverse effects in women with GDM in both the short term 


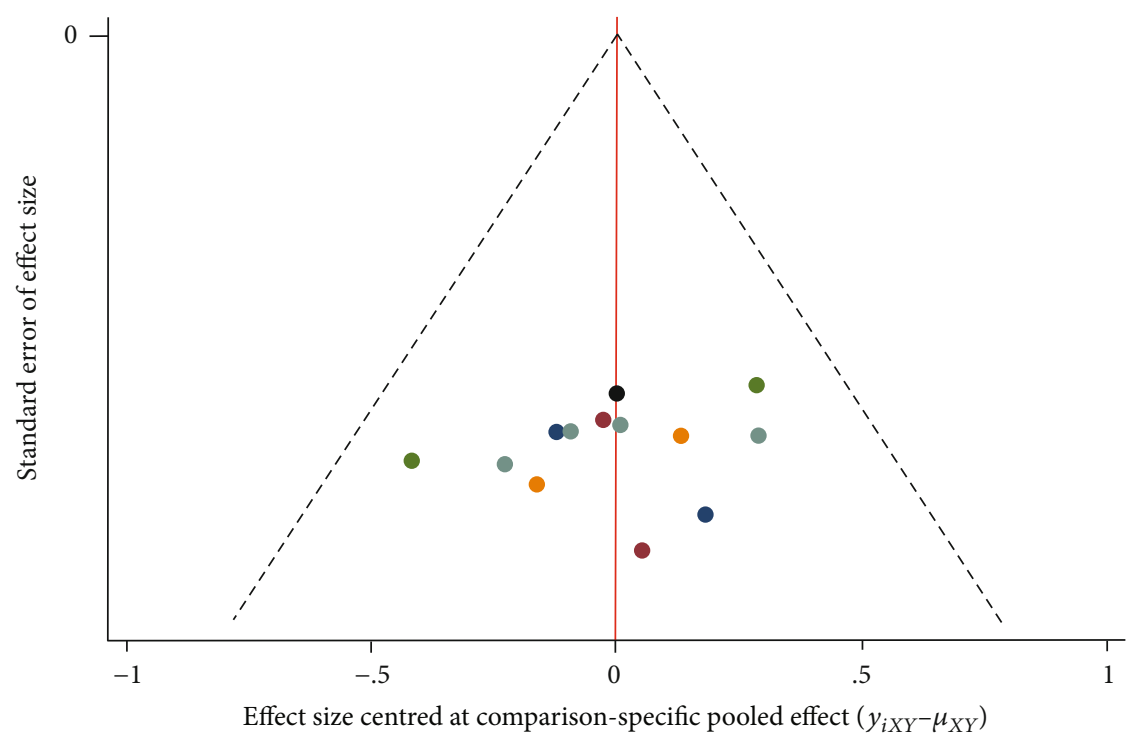

FIgURE 5: Comparison-adjusted funnel plot. Points of different colors represent different interventions.

and long term. It is essential to control blood glucose in women with GDM. Currently, nutritional management is a widely used method, and rational nutritional strategies can not only ensure the nutritional needs of mothers and infants but also effectively control blood glucose. Most pregnant women can achieve satisfactory blood glucose levels and good pregnancy outcomes through nutrition management [35]. An exploration of the effects of different types of nutritional supplementation on glucose metabolism indicated that such supplementation can not only be used as adjunctive therapies to nutritional treatment but also appropriately meet the nutritional needs of pregnant women and fetuses. At present, studies have shown the effectiveness of vitamin $\mathrm{D}$ and omega-3 [36-38] in improving glucose metabolism. Direct meta-analysis studies mostly analyze the effects of supplementation with a single nutrient compared with placebo, and few studies have compared the effects of different nutritional strategies. The effect of supplementation with different nutrients on glucose metabolism in GDM patients has not been uniformly assessed. Therefore, we searched for related published RCT studies, and NMA was used to perform direct and indirect comparisons of the impact of five nutritional supplementation strategies on FPG, serum insulin, and HOMA-IR in GDM patients. The effects were quantified to identify the best nutritional supplementation strategy to provide new ideas for adjunctive therapies in GDM patients.

In our study, pairwise meta-analysis and NMA results showed that nutrient supplementation significantly decreased FPG, serum insulin, and HOMA-IR compared with the effects of the placebo. According to the Academy of Nutrition and Dietetics, nutritional therapy is the basis of GDM therapy [39], and all individuals with prediabetes and any other type of diabetes should receive individualized nutritional therapy according to the condition. The NMA results showed that vitamin $\mathrm{D}$ is better than omega-3, zinc, and probiotics for decreasing FPG and that magnesium is better than probiotics for decreasing FPG. Vitamin D and magnesium have certain advantages compared with probiotics for decreasing serum insulin. Vitamin $\mathrm{D}$ has greater benefits for improving HOMA-IR than probiotics. There were no significant differences between the other nutrients that were supplemented.

According to the results of SUCRA, vitamin D supplementation was the best for reducing FPG and improving HOMA-IR compared with the effects of the other nutritional strategies, and magnesium supplementation ranked second. Vitamin D, also known as calciferol, is mainly active in the body as 25 -hydroxyvitamin $\mathrm{D}(25(\mathrm{OH}) \mathrm{D})$, which is often used as the best indicator for measuring vitamin D levels [40]. Vitamin D deficiency is a common phenomenon after pregnancy; a study showed that at 25-28 weeks of gestation, the concentration of $25(\mathrm{OH}) \mathrm{D}$ in GDM patients is significantly reduced [41]. Appropriate vitamin D supplementation to maintain optimal $25(\mathrm{OH}) \mathrm{D}$ levels is potentially beneficial for glucose metabolism. Vitamin D deficiency is considered a potential risk factor for abnormal glucose metabolism; Zhang et al. [42] conducted a study based on data free of the Hawthorne effect, and the study indicated that low levels of vitamin D in the blood may increase the risk of GDM and that appropriate vitamin $\mathrm{D}$ supplementation may improve GDM status. Studies have shown that vitamin D can stimulate the body to secrete insulin under physiological conditions [43] and that it is essential for maintaining normal glucose tolerance. $25(\mathrm{OH}) \mathrm{D}$ can not only regulate insulin secretion by binding to receptors in islet $\beta$ cells but also stimulate insulin receptor expression to promote insulin sensitivity [44], achieving the effect of decreasing blood glucose. In addition, vitamin $\mathrm{D}$ has antioxidant effects, which can reduce the damage to islet $\beta$ cells and the apoptosis of islet $\beta$ cells via active oxidative groups [45]. GDM patients can increase their concentration of $25(\mathrm{OH}) \mathrm{D}$ via vitamin D supplementation, thereby ameliorating insulin resistance and decreasing blood glucose [46]. 
According to the results of SUCRA, magnesium supplementation showed better results in terms of decreasing serum insulin, and in the cumulative ranking results, magnesium ranked first, followed by vitamin D. Magnesium can protect and repair islet $\beta$ cells [47]. Deficiency of magnesium can cause changes in the structure of pancreatic cells, reduce the particles in $\beta$ cells, and lead to insufficient insulin synthesis and secretion. Barbagallo et al. [48] showed that insufficient magnesium intake and lack of plasma magnesium can affect the process of glucose metabolism. $\mathrm{Mg}^{2+}$ is an important cation in cells and is a coenzyme involved in more than 300 enzymatic reactions [49]. Changes in magnesium concentration can affect islet responses, and $\mathrm{Mg}^{2+}$ deficiency is one of the nongenetic regulators of insulin resistance [50]. $\mathrm{Mg}^{2+}$ is regarded as a second messenger of insulin and plays an important role in the stability of glucose metabolism and insulin sensitivity. First, magnesium deficiency can reduce insulin receptor activity and result in insulin resistance. Second, hypomagnesemia inhibits glucose utilization in both basal and insulin-stimulated states [51]. Therefore, magnesium ions play an important role in glucose metabolism.

In summary, vitamin $\mathrm{D}$ and magnesium supplementation during pregnancy is more effective than supplementation with other nutrients for women with GDM. To the best of our knowledge, this is the first NMA comparing the effects of different nutritional strategies in maintaining glucose metabolic homeostasis. A particular advantage of NMA is that one can estimate the effects among different interventions by incorporating direct and indirect evidence. However, this review also has some limitations. First, we included thirteen studies; however, most of the studies included in our review are from Iran. Subjects were requested to not change their routine physical activity or usual dietary intake throughout the study; however, every country has different eating habits and routines for pregnancy care and medications that may cause differences in regulating glucose homeostasis in different countries, which may introduce uncertainty in clinical significance and influence the universality of outcomes. Moreover, some of the studies had fewer samples, and some studies had a high risk bias due to the lack of allocation concealment and blinding of outcome assessment, requiring more relevant high-quality and large-scale studies in the future. Second, the number of studies included in each intervention is limited. There are differences in the inclusion criteria for GDM and the types and doses of nutritional supplements in each study that lead to increased heterogeneity; therefore, more studies and well-controlled design are needed to decrease heterogeneity and provide more evidence. Third, because our subjects were women with GDM, the majority of the intervention durations in the studies were approximately six weeks, which may have affected the conclusion. Fourth, most of our studies were placebo-controlled trials; the number of head-to-head trials that directly compared different nutritional supplement strategies was limited, and more direct evidence of different nutritional strategies is needed to further validate our conclusions in the future.

\section{Conclusions}

Overall, vitamin D intake has a significant effect of reducing FPG and improving HOMA-IR. Magnesium intake has a superior effect of regulating serum insulin than supplementation with other nutrients. In addition, the results of our study indicate that omega-3, zinc, and probiotic supplementation are beneficial for maintaining glucose homeostasis. The present results suggest that these nutritional supplements may be considered adjunctive therapies for glycemic control in women with GDM. However, the limitations of the study may affect the clinical significance and universality of the results. Further studies are warranted to reduce the limitations of the existing evidence and to confirm the above conclusions.

\section{Conflicts of Interest}

The authors declare that they have no competing interests.

\section{Authors' Contributions}

SJ, LS, JD, and JY conceived and designed the research. SJ, LS, and JD retrieved the literature and identified eligible studies. SJ and JD extracted the data. SJ, LS, and JY analyzed the data. SJ, YL, and XG checked the statistical methods. SJ, LS, JD, JY, and $\mathrm{BH}$ interpreted the data. SJ wrote the first draft. SJ, LS, JY, YL, and XG reviewed the manuscript and revised the important content. All authors read and approved the final draft.

\section{Supplementary Materials}

Annex 1: detailed search strategy. (Supplementary Materials)

\section{References}

[1] The American Diabetes Association, "Diagnosis and classification of diabetes mellitus," Diabetes Care, vol. 37, Supplement 1, pp. S81-S90, 2014.

[2] D. A. Sacks, D. R. Coustan, D. R. Hadden et al., "Frequency of gestational diabetes mellitus at collaborating centers based on IADPSG consensus panel-recommended criteria: the Hyperglycemia and Adverse Pregnancy Outcome (HAPO) study," Diabetes Care, vol. 35, no. 3, pp. 526-528, 2012.

[3] Z. Groof, G. Garashi, H. Husain et al., "Prevalence, risk factors, and fetomaternal outcomes of gestational diabetes mellitus in Kuwait: a cross-sectional study," Journal of Diabetes Research, vol. 2019, Article ID 9136250, 7 pages, 2019.

[4] L. Hartling, D. M. Dryden, A. Guthrie, M. Muise, B. Vandermeer, and L. Donovan, "Benefits and harms of treating gestational diabetes mellitus: a systematic review and meta-analysis for the U.S. Preventive Services Task Force and the National Institutes of Health Office of Medical Applications of Research," Annals of Internal Medicine, vol. 159, no. 2, pp. 123-129, 2013.

[5] U. M. Schafer-Graf, U. Gembruch, F. Kainer et al., "Gestational diabetes mellitus (GDM) - diagnosis, treatment and follow-up. Guideline of the DDG and DGGG (S3 level, AWMF registry number 057/008, February 2018)," Geburtshilfe und Frauenheilkunde, vol. 78, no. 12, pp. 1219-1231, 2018. 
[6] U. Andersson-Hall, C. Gustavsson, A. Pedersen, D. Malmodin, L. Joelsson, and A. Holmang, "Higher concentrations of BCAAs and 3-HIB are associated with insulin resistance in the transition from gestational diabetes to type 2 diabetes," Journal Diabetes Research, vol. 2018, article 4207067, 12 pages, 2018.

[7] T. L. Hernandez and J. C. Brand-Miller, "Nutrition therapy in gestational diabetes mellitus: time to move forward," Diabetes Care, vol. 41, no. 7, pp. 1343-1345, 2018.

[8] T. A. Buchanan, A. H. Xiang, and K. A. Page, "Gestational diabetes mellitus: risks and management during and after pregnancy," Nature Reviews Endocrinology, vol. 8, no. 11, pp. 639-649, 2012.

[9] T. L. Hernandez, R. E. Van Pelt, M. A. Anderson et al., "Women with gestational diabetes mellitus randomized to a higher-complex carbohydrate/low-fat diet manifest lower adipose tissue insulin resistance, inflammation, glucose, and free fatty acids: a pilot study," Diabetes Care, vol. 39, no. 1, pp. 39-42, 2016.

[10] M. Hod, A. Kapur, D. A. Sacks et al., "The International Federation of Gynecology and Obstetrics (FIGO) initiative on gestational diabetes mellitus: a pragmatic guide for diagnosis, management, and care," International Journal of Gynaecology and Obstetrics, vol. 131, no. S3, pp. S173-S211, 2015.

[11] American Diabetes Association, "14. Management of diabetes in Pregnancy: Standards of medical care in diabetes-2019," Diabetes Care, vol. 42, Supplement 1, pp. S165-S172, 2019.

[12] D. Thompson, H. Berger, D. Feig et al., "Diabetes and pregnancy," Canadian Journal of Diabetes, vol. 37, Supplement 1, pp. S168-S183, 2013.

[13] M. Haneda, M. Noda, H. Origasa et al., "Japanese clinical practice guideline for diabetes 2016," Journal of Diabetes Investigation, vol. 9, no. 3, pp. 657-697, 2018.

[14] T. L. Hernandez, M. A. Anderson, C. Chartier-Logan, J. E. Friedman, and L. A. Barbour, "Strategies in the nutritional management of gestational diabetes," Clinical Obstetrics and Gynecology, vol. 56, no. 4, pp. 803-815, 2013.

[15] B. Hutton, G. Salanti, D. M. Caldwell et al., "The PRISMA extension statement for reporting of systematic reviews incorporating network meta-analyses of health care interventions: checklist and explanations," Annals of Internal Medicine, vol. 162, no. 11, pp. 777-784, 2015.

[16] A. Liberati, D. G. Altman, J. Tetzlaff et al., "The PRISMA statement for reporting systematic reviews and meta-analyses of studies that evaluate healthcare interventions: explanation and elaboration," BMJ, vol. 339, article b2700, 2009.

[17] J. P. Higgins, D. G. Altman, P. C. Gotzsche et al., "The Cochrane Collaboration's tool for assessing risk of bias in randomised trials," BMJ, vol. 343, no. 2, article d5928, 2011.

[18] J. P. Higgins, S. G. Thompson, J. J. Deeks, and D. G. Altman, "Measuring inconsistency in meta-analyses," BMJ, vol. 327, no. 7414, pp. 557-560, 2003.

[19] A. C. Tricco, H. M. Ashoor, J. Antony et al., "Safety, effectiveness, and cost effectiveness of long acting versus intermediate acting insulin for patients with type 1 diabetes: systematic review and network meta-analysis," BMJ, vol. 349, no. 6, article g5459, 2014.

[20] J. P. Jansen and H. Naci, "Is network meta-analysis as valid as standard pairwise meta-analysis? It all depends on the distribution of effect modifiers," BMC Medicine, vol. 11, no. 1, p. 159, 2013.
[21] S. P. Hozo, B. Djulbegovic, and I. Hozo, "Estimating the mean and variance from the median, range, and the size of a sample," BMC Medical Research Methodology, vol. 5, p. 13, 2005.

[22] M. Jamilian, M. Samimi, N. Mirhosseini et al., "A randomized double-blinded, placebo-controlled trial investigating the effect of fish oil supplementation on gene expression related to insulin action, blood lipids, and inflammation in gestational diabetes mellitus-fish oil supplementation and gestational diabetes," Nutrients, vol. 10, no. 2, p. 163, 2018.

[23] M. Samimi, M. Jamilian, Z. Asemi, and A. Esmaillzadeh, "Effects of omega-3 fatty acid supplementation on insulin metabolism and lipid profiles in gestational diabetes: randomized, double-blind, placebo-controlled trial," Clinical Nutrition, vol. 34, no. 3, pp. 388-393, 2015.

[24] M. Jamilian, M. Samimi, F. A. Ebrahimi et al., "The effects of vitamin D and omega-3 fatty acid co-supplementation on glycemic control and lipid concentrations in patients with gestational diabetes," Journal of Clinical Lipidology, vol. 11, no. 2, pp. 459-468, 2017.

[25] Z. Asemi, M. Karamali, M. Jamilian et al., "Magnesium supplementation affects metabolic status and pregnancy outcomes in gestational diabetes: a randomized, double-blind, placebocontrolled trial," The American Journal of Clinical Nutrition, vol. 102, no. 1, pp. 222-229, 2015.

[26] M. Jamilian, M. Samimi, A. E. Faraneh et al., "Magnesium supplementation affects gene expression related to insulin and lipid in patients with gestational diabetes," Magnesium Research, vol. 30, no. 3, pp. 71-79, 2017.

[27] Z. Asemi, T. Hashemi, M. Karamali, M. Samimi, and A. Esmaillzadeh, "Effects of vitamin D supplementation on glucose metabolism, lipid concentrations, inflammation, and oxidative stress in gestational diabetes: a double-blind randomized controlled clinical trial," The American Journal of Clinical Nutrition, vol. 98, no. 6, pp. 1425-1432, 2013.

[28] Q. Li and B. Xing, "Vitamin D3-supplemented yogurt drink improves insulin resistance and lipid profiles in women with gestational diabetes mellitus: a randomized double blinded clinical trial," Annals of Nutrition \& Metabolism, vol. 68, no. 4, pp. 285-290, 2016.

[29] M. Karamali, Z. Heidarzadeh, S. M. Seifati et al., "Zinc supplementation and the effects on metabolic status in gestational diabetes: a randomized, double-blind, placebo-controlled trial," Journal of Diabetes and its Complications, vol. 29, no. 8, pp. 1314-1319, 2015.

[30] N. Roshanravan, M. Alizadeh, M. Hedayati et al., "Effect of zinc supplementation on insulin resistance, energy and macronutrients intakes in pregnant women with impaired glucose tolerance," Iranian Journal of Public Health, vol. 44, no. 2, pp. 211-217, 2015.

[31] M. Babadi, A. Khorshidi, E. Aghadavood et al., "The effects of probiotic supplementation on genetic and metabolic profiles in patients with gestational diabetes mellitus: a randomized, double-blind, placebo-controlled trial," Probiotics Antimicrob Proteins, vol. 11, no. 4, pp. 1227-1235, 2018.

[32] B. Badehnoosh, M. Karamali, M. Zarrati et al., "The effects of probiotic supplementation on biomarkers of inflammation, oxidative stress and pregnancy outcomes in gestational diabetes," The Journal of Maternal-Fetal \& Neonatal Medicine, vol. 31, no. 9, pp. 1128-1136, 2018.

[33] M. Karamali, F. Dadkhah, M. Sadrkhanlou et al., "Effects of probiotic supplementation on glycaemic control and lipid profiles in gestational diabetes: a randomized, double-blind, 
placebo-controlled trial," Diabetes \& Metabolism, vol. 42, no. 4, pp. 234-241, 2016.

[34] A. Kijmanawat, P. Panburana, and S. Reutrakul, "Effects of probiotic supplements on insulin resistance in gestational diabetes mellitus: a double-blind randomized controlled trial," Journal of Diabetes Investigation, vol. 10, no. 1, pp. 163-170, 2019.

[35] E. Tsirou, M. G. Grammatikopoulou, X. Theodoridis et al., "Guidelines for medical nutrition therapy in gestational diabetes mellitus: systematic review and critical appraisal," Journal of the Academy of Nutrition and Dietetics, vol. 119, no. 8, pp. 1320-1339, 2019.

[36] O. Ojo, S. M. Weldon, and T. Thompson, "The effect of vitamin D supplementation on glycaemic control in women with gestational diabetes mellitus: a systematic review and metaanalysis of randomised controlled trials," International Journal of Environmental Research and Public Health, vol. 16, no. 10, p. 1716, 2019.

[37] R. Sahebi, M. Rezayi, M. Emadzadeh et al., "The effects of vitamin D supplementation on indices of glycemic control in Iranian diabetics: a systematic review and meta-analysis," Complementary Therapies in Clinical Practice, vol. 34, pp. 294-304, 2019.

[38] N. Zhong and J. Wang, "The efficacy of omega-3 fatty acid for gestational diabetes: a meta-analysis of randomized controlled trials," Gynecological Endocrinology, vol. 35, no. 1, pp. 4-9, 2019.

[39] M. O. Duarte-Gardea, D. M. Gonzales-Pacheco, D. M. Reader et al., "Academy of nutrition and dietetics gestational diabetes evidence-based nutrition practice guideline," Journal of the Academy of Nutrition and Dietetics, vol. 118, no. 9, pp. 17191742, 2018.

[40] A. Munoz-Garach, B. Garcia-Fontana, and M. Munoz-Torres, "Vitamin D status, calcium intake and risk of developing type 2 diabetes: an unresolved issue," Nutrients, vol. 11, no. 3, p. 642, 2019.

[41] S. Soheilykhah, M. Mojibian, M. Rashidi, S. Rahimi-Saghand, and F. Jafari, "Maternal vitamin D status in gestational diabetes mellitus," Nutrition in Clinical Practice, vol. 25, no. 5, pp. 524-527, 2010.

[42] Y. Zhang, Y. Gong, H. Xue, J. Xiong, and G. Cheng, "Vitamin $\mathrm{D}$ and gestational diabetes mellitus: a systematic review based on data free of Hawthorne effect," BJOG, vol. 125, no. 7, pp. 784-793, 2018.

[43] J. A. Alvarez and A. Ashraf, "Role of vitamin D in insulin secretion and insulin sensitivity for glucose homeostasis," International Journal of Endocrinology, vol. 2010, Article ID 351385, 18 pages, 2010.

[44] A. Vaidya and J. S. Williams, "Vitamin D and insulin sensitivity: can gene association and pharmacogenetic studies of the vitamin D receptor provide clarity?," Metabolism, vol. 61, no. 6, pp. 759-761, 2012.

[45] M. Lu, Y. Xu, L. Lv, and M. Zhang, "Association between vitamin D status and the risk of gestational diabetes mellitus: a meta-analysis," Archives of Gynecology and Obstetrics, vol. 293, no. 5, pp. 959-966, 2016.

[46] Y. Chen, B. Zhu, X. Wu, S. Li, and F. Tao, "Association between maternal vitamin $\mathrm{D}$ deficiency and small for gestational age: evidence from a meta-analysis of prospective cohort studies," BMJ Open, vol. 7, no. 8, article e016404, 2017.
[47] T. Gunther, "The biochemical function of $\mathrm{Mg}^{2}+$ in insulin secretion, insulin signal transduction and insulin resistance," Magnesium Research, vol. 23, no. 1, pp. 5-18, 2010.

[48] M. Barbagallo, G. Di Bella, V. Brucato et al., "Serum ionized magnesium in diabetic older persons," Metabolism, vol. 63, no. 4, pp. 502-509, 2014.

[49] J. Takaya, F. Yamato, Y. Kuroyanagi, H. Higashino, and K. Kaneko, "Intracellular magnesium of obese and type 2 diabetes mellitus children," Diabetes Therapy, vol. 1, no. 1, pp. 25-31, 2010.

[50] H. Rosolova, O. Mayer Jr., and G. M. Reaven, "Insulin-mediated glucose disposal is decreased in normal subjects with relatively low plasma magnesium concentrations," Metabolism, vol. 49, no. 3, pp. 418-420, 2000.

[51] L. E. Simental-Mendia, M. Rodriguez-Moran, and F. Guerrero-Romero, "Failure of $\beta$-cell function for compensate variation in insulin sensitivity in hypomagnesemic subjects," Magnesium Research, vol. 22, no. 3, pp. 151-156, 2009. 